\title{
Erratum: Neutron phase spin echo [Phys. Rev. C 93, 045501 (2016)]
}

\author{
Florian M. Piegsa, Patrick Hautle, and Christian Schanzer
}

(Received 23 May 2016; published 16 June 2016)

DOI: 10.1103/PhysRevC.93.069903

This Erratum is meant to clarify the conclusions of the original article by providing more details concerning the static magnetic field configuration applied in the neutron phase spin echo method. The measurements and results presented in the article are not affected. However, to be able to measure changes of the kinetic energy with the given resolution, the magnetic field of the apparatus has to be adapted.

The technique has been described with a homogeneous magnetic field $B_{0}$ applied along the $z$ axis in which the three spin-flip coils are positioned. This matches the actual configuration of the employed experimental setup. Using such a field allows for observing phase shifts induced by magnetic or pseudomagnetic interactions of the neutron spin. This was demonstrated using an auxiliary coil positioned in one of the spectrometer arms; compare with Fig. 4(b) of the original article. The situation corresponds also to most of the stated fundamental neutron physics applications, e.g., the search for an electric dipole moment of the neutron, exotic interactions, parity-violating spin rotations, etc. For experiments intended to measure a change in the kinetic energy, a different magnetic field configuration is required. In this case a phase shift is only obtained if the magnetic field $B_{0}$ is applied at the positions of the spin-flip coils, whereas the field between them is reduced to a value close to zero. This is the identical configuration as in the cited Ref. [1] and as briefly mentioned in the first footnote of the original article. As a consequence, the attainable energy resolution $\Delta E$ obtained from the measured phase stability (compare with Fig. 3) has to be interpreted as a projected sensitivity. The associated field settings for the two cases are presented in the additional figure (Fig. 5, not shown in the original article).

The authors would like to acknowledge important discussions with O. Zimmer.

(a)

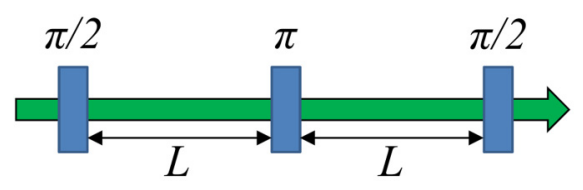

(b)

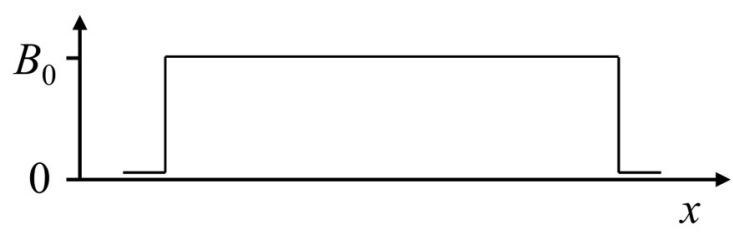

(c)

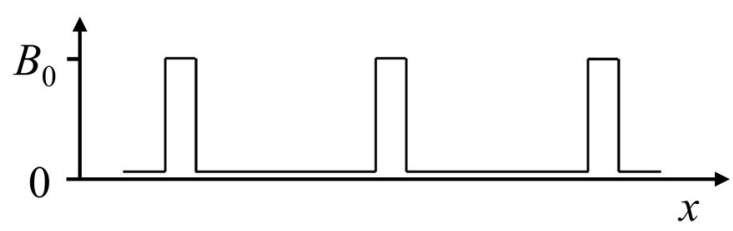

FIG. 5. (a) Scheme of the neutron phase spin echo setup with the three spin-flip coils separated by a distance $L$ and the neutron beam in the $x$ direction. (b) Static magnetic field configuration with a homogeneous field $B_{0}$ along the $z$ axis to measure magnetic and pseudomagnetic effects acting on the neutron spin. This setting was applied in the presented experimental apparatus. (c) Dedicated field configuration to measure inelastic effects, i.e., changes in the kinetic energy. 